\title{
Género, clasificación y nombres ligados en tikuna (Amazonia colombiana)
}

\author{
Gender, classification and linked nouns in tikuna
}

María Emilia Montes Rodríguez ${ }^{1}$

\begin{abstract}
Resumen
La presencia de sistemas de clasificación nominal relativamente complejos, es un rasgo difundido en la Amazonia, pero no homogéneo, pues hay importantes variaciones tipológicas entre las lenguas y las familias involucradas. El entorno regional tikuna es un entorno de clasificación. El grado de codificación gramatical difiere y es por ello un factor importante en la mirada areal y tipológica. Proponemos aquí que el tikuna expresa un punto dentro del continuum regional: a) separa género de clasificadores y b) los clasificadores son elementos léxicos plenos, con gramaticalización muy parcial (¿incipiente?). En otras lenguas del área las clases nominales instauran una concordancia obligatoria en el nivel de la oración y del sintagma nominal. Lo que subyace a la clasificación de los nominales -expresada por estos sistemas morfosintácticos de clasificación- es tanto una visión del mundo mediada por categorías y experiencias de la cultura, como una expresión de universales semánticos.
\end{abstract}

\begin{abstract}
The presence of relatively complex systems of nominal classification, is a feature spread in the Amazon, but this is not an homogeneous fact, as there are important typological variations between languages and the families involved. The tikuna regional environment is an environment of classification. The degree of grammatical encoding differs between languages and is therefore an important factor for areal and typological look. We propose here that the tikuna expresses a point within the regional continuum: a) it separates gender from classifiers b) classifiers are full lexical items with (initial?) only partial grammaticalization. In other languages of this area noun classes establish agreement at the level of the sentence and in the noun phrase. What underlies the classification of nominals -expressed by these morphosyntactic classification systems- is both a worldview mediated by experiences and categories of culture, as well as an expression of universal semantic.
\end{abstract}

\section{Introducción}

Todas las lenguas tienen formas gramaticales o léxicas para categorizar referentes, sea a partir de la cantidad, o de los rasgos humano y animado, o a partir del tamaño y de la agrupación, o de la mensurabilidad, o de la función, la materia o la configuración. Aquello que llamamos clasificadores remite a sistemas más rígidamente estructurados, exhaustivos y por ello susceptibles de

\footnotetext{
${ }^{1}$ Profesora asociada del Departamento de Lingüística, Facultad de Ciencias Humanas de la Universidad Nacional de Colombia. Correo-e: memontesr@unal.edu.co.
} 
revelar mejor "los esquemas especialmente salientes de las visiones del mundo de sus hablantes"2. Las lenguas africanas de la familia Bantú son un ejemplo clásico -y bien estudiado- de la existencia de un sistema extenso y complejo de géneros, clases nominales o clasificadores. La lengua dyirbal y otras lenguas de Australia, lenguas del sureste asiático, lenguas centro y norteamericanas están abundantemente referenciadas en la literatura científica sobre el tema, sea desde la óptica de los estudios etnolingüísticos y cognitivos, sea desde la óptica gramatical y morfosintáctica.

El contenido de los clasificadores no es fácilmente predecible desde fuera, sin acceso a la pragmática y a la cultura. Para las clases nominales de la lengua andoke (que se manifiestan junto con la modalidad cognitiva y el tiempo bajo la forma de índices referenciales de tercera persona, dentro del asertivo) se establecen los siguientes valores semánticos para las seis clases posibles:

(...) entidades no animadas, poco individualizadas, substancias, términos abstractos, algunos artefactos, numerosas plantas, ríos, etc. (...); árboles leñosos, objetos rígidos, huesos, entidades asociadas a la idea de rigidez y de palo (...); algunos artefactos (distintos de los dos anteriores), elementos vegetales como semillas, frutas, hongos, palmeras y de árboles no leñosos; entidades animadas consideradas como masculinas (la mayoría de especies animales, los varones, los astros, etc.) (...); entidades animadas consideradas como femeninas (las otras especies animales, como por ejemplo algunas culebras, murciélagos, pájaros, ratones, etc., las mujeres) (...); entidades animadas consideradas como colectivas (los grupos humanos, algunos grupos de animales, un difunto, una mujer en edad de procrear, etc. $)^{3}$

Algunos hechos que podrían verse como efectos de una clasificación arbitraria, análoga a la del género en lenguas romances, se aclaran con la explicación cultural y ambiental que establece nexos entre los elementos que están dentro de una clase.

La lengua tikuna no es un sistema típico de clasificadores. En nuestra presentación hemos considerado fundamental presentar hechos que describen el grado de gramaticalización de los posibles clasificadores con respecto a un polo léxico; así como una comparación con los datos sobre género (éste sí gramaticalizado y separado de la clasificación) y finalmente la interpretación sobre las funciones que cumpliría la clasificación nominal en esta lengua, con algunos comentarios iniciales sobre su posible lugar en el continuum

\footnotetext{
${ }^{2}$ Palmer, Gary. Lingüistica cultural. (Madrid: Alianza Editorial, 2000), 158.

${ }^{3}$ Landaburu, Jon. "La lengua andoke." En Lenguas indígenas de Colombia. Una visión descriptiva, editado por González, M. Stella y Rodríguez, M. Luisa. (Bogotá: Instituto Caro y Cuervo, 2000), 278.
} 
de clasificación del Noroeste Amazónico. En cuanto a la semántica de la clasificación, vemos que hay coincidencias - en el contenido- con otras lenguas de la región descritas como poseedoras de sistemas típicos de clasificación nominal (necesidad de distinguir entre animado e inanimado, especificidad de los colectivos, términos abstractos para formas tubulares, alargadas, filiformes, compactas, laminares; términos para sustancia, materia y agrupación; valor individualizante y singularizador del clasificador vs. el nombre genérico, entre muchos otros rasgos semánticos posibles). También destacamos la convergencia con otros estudios que señalan la importancia de la taxonomía y la denominación de partes de los vegetales para una mejor comprensión de la semántica de la clasificación nominal.

\section{Tikuna, pueblo en contacto}

Los tikunas son una población que habita principalmente en la orilla izquierda del río Amazonas y sus afluentes, en territorios peruanos, colombianos y brasileros. La etimología de tikuna (tükuna, tekuna, tukuna, tokuna) es incierta. Recientemente se estableció la filiación del tikuna con el yurí reportado por Martius ${ }^{4}$, así que la lengua tikuna de hoy sería la heredera de un complejo lingüístico (y cultural) antes de mayor extensión, que abarcaba dos grandes áreas interfluviales en el Noroeste Amazónico: Amazonas-Putumayo y PutumayoCaquetás.

La región donde aparecen ubicados los yurí y los tikunas en las crónicas es también un área Arawak de lenguas de grupos desaparecidos tempranamente: uainumá, yumaná, passé, mariaté, kauyxana (kaiueschana), o recientemente: resígaro, tariana. Sitios históricos de los yurí estaban próximos de los territorios uitotos, boras y andokes (complejo cultural de la 'Gente de centro') muy numerosos y dinámicos antes del etnocidio cauchero de finales del siglo XIX y principios del siglo XX. La presencia Caribe (hubo gente carijona en la región) fue importante. Los Tupí llegaron por el Amazonas-Solimões en las invasiones previas a la conquista. La lingua geral o ñe'engatú, fue difundida por las misiones y dejó una impronta muy visible en toda la región.

A la llegada de los conquistadores los tikunas estaban en la tierra firme en cabeceras de los afluentes del Amazonas; fabricaba un curare (veneno paralizante) muy reconocido y eran enemigos de los poderosos omaguas de la ribera y las islas del Amazonas. Mantuvieron contactos con los yaguas y con los mayorunas

\footnotetext{
${ }^{4}$ Carvalho, Fernando Orphão de "On the genetic kinship of the languages Tikúna and Yurí", Revista Brasileira de Linguística Antropológica 1 (2), 2009.

${ }^{5}$ Goulard, Jean Pierre y Montes, María Emilia “Los Yurí/Juri-Tikuna, en el complejo sociolingüistico del noroeste amazónico" LIAMES Linguas Indígenas Americanas, vol. 13, 2013.
} 
(y quizá con otras gentes del grupo Pano). Hoy son una población numerosa y bastante vital, que parece incorporar rápidamente los procesos de cambio cultural y socioeconómico. En Colombia hay avanzados grados de bilingüismo y de pérdida de la lengua nativa. Sumando la población de Brasil, Colombia y Perú, habría entre treinta y cuarenta mil personas. Habitan un territorio continuo en una zona donde hay importantes centros urbanos como Iquitos, Leticia, Saõ Paulo de Olivença y Manaus. En Colombia los tikunas ribereños comparten su territorio con los cocamas que ocupa las islas amazónicas, con los yaguas y con otros grupos indígenas que han migrado recientemente a la ribera amazónica como los uitotos.

Alguna vez se consideró a la lengua tikuna como lengua Arawak ${ }^{6}$, idea que Nimuendaju descartó. Este autor le atribuye influencias Je y Pano por verificar ${ }^{7}$. Como otras lenguas de la gran región amazónica es una lengua tonal, con un sistema de vocales orales/nasales similar al de las lenguas Tukano; en su vocabulario se detectan fácilmente préstamos Tupí y algunos quechuismos. Está dialectalizada y no hay estudios suficientes sobre los dialectos más aislados de la 'tierra firme' o zona de refugio interfluvial. Hay descripciones fonológicas y morfosintácticas hechas sobre puntos geográficos precisos en las riberas de Perú $^{8}$, Brasil $^{9}$, Colombia $^{10}$.

Según propuesta en uso en la ribera colombiana, utilizamos una ortografía en la se postulan 6 vocales orales: a, e, i, o, u, ü y 6 vocales nasales, ã ẽ ĩ õ ũ ü. La vocal representada ortográficamente por ü corresponde a /u/, una vocal deslabializada, posterior, alta. La nasalidad es silábica y se indica sobre la vocal (ã) o en la consonante: $\mathrm{m}, \mathrm{n}, \tilde{n}$, ng (alófonos de oclusivas sonoras labial, alveolar, palatal y velar respectivamente). En dialectos de tierra firme, Trapecio Amazónico colombiano, se proponen tres tonos fonémicos (Alto, Medio, Bajo). Los tonos alto y bajo se marcan con acento agudo y grave respectivamente (á, à). La longitud vocálica se indica con dos puntos al lado de la voca (a:); éste es un rasgo prosódico de morfemas léxicos y parece debilitarse en dialectos ribereños orientales. La entonación se puede manifestar en terrazas tonales (descensos o ascensos generalizados de una porción del enunciado) cuando hay dislocamientos o focalización. Estos hechos se señalan antes del segmento

\footnotetext{
${ }^{6}$ Rivet, Paul. "Affinités du Tikuna”. Journal de la Societé des Américanistes. Tomo IX. (Paris, 1912).

${ }^{7}$ Nimuendaju, Kurt. The Tukuna. (Berkeley: University of California Press, 1952):156-158.

${ }^{8}$ Anderson, Lambert. "The structure and distribution of the ticuna independent clause". Lingustics 20. (Paris: Mouton, 1966).

${ }^{9}$ Soares-Facó, Marilia. Investigação de aspectos da sintaxe Tikuna: O supra-segmental em Tikuna e a teoria fonologica. Vol. I. (Campinas: Editora da UNICAMP, 2000).

${ }^{10}$ Montes Rodríguez, M. Emilia. Morfosintaxis de la lengua tikuna. (Bogotá: Universidad de Los Andes-CESO-CCELA, 2004).
} 
afectado (' o '). Los tonos se indican también con letras mayúsculas para los tonemas A, M, B; y minúsculas para los alótonos (por ejemplo, $a b$ para 'altobajo' o modulado descendente, $a$ ' para alto downstep).

\section{Género tripartito, valores temporales y modales asociados}

El género es la categoría más visible que se expresa en todos los nombres; constituye un sistema independiente de los clasificadores, se expresa a través de un paradigma de tres formas que se anteponen a los nombres o a los sintagmas nominales en ciertos contextos gramaticales. Los morfemas son $\{\mathrm{i}\},\{\mathrm{ya}\}$ (éste es $\{a\}$ en los dialectos de la ribera hacia el oriente) y $\{\mathrm{ga}\}$. En el presente los morfemas $\{\mathrm{i}\}$, \{ya $\}$ expresan la oposición entre dos clases de nombres (masculino/femenino). En el pasado (o caducidad, categoría que incluye valores modales) masculino y femenino se unifican en $\{\mathrm{ga}\}$. El género cubre todos los nombres, tanto con referentes animados como inanimados. También los conectores y los demostrativos tienen elementos que diferencian género y tiempo-modalidad. La diferencia entre animado/inanimado se expresa sólo en un grupo de demostrativos ilustrados aquí (ver ejemplos del cuadro 4, infra). Este rasgo de caducidad parece original del sistema tikuna, pues lo común en otras lenguas amazónicas es tener un sistema tripartito (masculino y femenino que se unifican en el plural para los animados) sin valores temporales-modales.

En tikuna el género y los mecanismos de clasificación nominal son dos sistemas separados. El primero de ellos está altamente gramaticalizado $\mathrm{y}$ es exhaustivo. Tiene una base biológica que justifica el uso de los rótulos 'femenino' y 'masculino' pero puede considerarse que la asignación de género a inanimados y seres no sexuados es generalmente arbitraria, similar a lo que ocurre en lenguas con sistemas típicos de género. La clasificación nominal se realiza de manera opcional, dependiendo de factores pragmáticos y textuales. Se expresa en construcciones en las que aparece un subconjunto de nombres ligados (que son nombres plenos) con valor semántico genérico. Por no ser un paradigma gramatical (hay indicios muy parciales de gramaticalización), no hablaremos de clasificadores en sentido estricto, pero sí de clasificación nominal como efecto resultante de la determinación y composición con estos nombres.

Resumimos las características de estos dos sistemas:

Cuadro 1: Comparación del género y de la clasificación en tikuna

\begin{tabular}{|l|c|l|}
\hline & Género & $\begin{array}{l}\text { Clasificación con } \\
\text { nombres ligados }\end{array}$ \\
\hline 1. Gramaticalización & SÍ & NO (parcial) \\
\hline 2. Obligatoriedad & SÍ & NO (pragmática) \\
\hline
\end{tabular}




\begin{tabular}{|l|c|l|}
\hline 3. Exhaustividad & Sí & NO \\
\hline 4. Función de derivación & NO & SÍ \\
\hline 5. Instaura concordancia en el nivel oracional & Sí & NO (anáfora) \\
\hline $\begin{array}{l}\text { 6. Instaura concordancia en el nivel del sintagma } \\
\text { nominal }\end{array}$ & Sí & NO (singulariza) \\
\hline $\begin{array}{l}\text { 7. Cumple funciones de individualización/ } \\
\text { singularización/definición/ anáfora/ rastreo de } \\
\text { la referencia }\end{array}$ & SÍ & SÍ \\
\hline
\end{tabular}

Una primera manifestación del género (masculino/femenino) es visible en las formas personales de tercera persona singular; en plural la distinción desaparece:

\section{Cuadro 2a: Género en las formas personales ligadas de tercera persona (prefijos de nombre o verbo)}

\begin{tabular}{|l|l|c|l|}
\hline & $\begin{array}{c}\text { Índices, prefijos } \\
\text { del verbo }\end{array}$ & Tonos & \multicolumn{1}{c|}{ Ejemplos } \\
\hline 3PM & na-, ni- & M & nã-nẽ "hijo de él" \\
\hline 3PF & ngĩ-, ĩ- & A & ngĩ-nẽ, ĩ- nẽ "hijo de ella" \\
\hline 3PPPL & tá-, tí- & A & tá-nẽ "hijo de ellos, ellas" \\
\hline 3P & tüma- & MM & $\begin{array}{l}\text { tümã-nẽ "hijo de él, ella, ellos o ellas } \\
\text { (respetuoso o ritual si el referente es singular)" }\end{array}$ \\
\hline
\end{tabular}

Cuadro 2b: Género en las formas personales libres de tercera persona

\begin{tabular}{|l|l|l|c|}
\hline \multicolumn{3}{|c|}{ Pronombres libres } & Tonos \\
\hline 3PM & nüma & "él" & $\mathrm{MM}$ \\
\hline 3PF & ngî́ma & "ella" & $\mathrm{AB}$ \\
\hline 3P & tüma & "ella, él, ellos, ellas" & $\mathrm{MM}$ \\
\hline
\end{tabular}

El género es una categoría encubierta, no tiene expresión morfológica en el nombre. El sintagma nominal objeto en la forma canónica es preverbal y allí no es evidente la manifestación de la marca de género:

tứè chà -tò: "siembro yuca" yuca $1 \mathrm{PS}^{11}$-sembrar

11 1PP Primera persona plural, 1PS Primera persona singular, 2PS Segunda persona singular, 3P Tercera persona, 3PFS Tercera persona femenina singular, 3PMS Tercera persona masculino singular, AC Acusativo, AN Animado, ATE Atenuativo, aspecto, BP 
Si el sintagma nominal objeto es postverbal, el género se manifiesta con uno de los tres proclíticos: $\{y a\}$ masculino, $\{i\}$ femenino en el presente; $\{\mathrm{ga}\}$ forma única del pasado.

chà-tò: ya tǘè "siembro yuca"

1PS sembrar G yuca

marü chà-na-mù i kowí "ya chupé bacur'”" (fruto silvestre similar al madroño) ya 1PS-O INT-chupar G bacurí

airú ya wàékü í na-ngò: i bú:kü "el perro negro mordió a la niña" perro G negro M 3PMS-morder G niña

El sintagma nominal Sujeto en oraciones focalizadas también recibe la marca de género en algunos casos (en este caso un pronombre introducido por el proclítico de femenino dicho por un locutor masculino). El femenino es el género no marcado.

kù-ữ chà-ngù:-ẽế niî́ 'chaù-gà' i chòmà "a tí es que enseño mi idioma, yo" 2PS-AC 1PS-aprender-CAU CÓP+3P 1PS-idioma G 1PS+BP

El género instaura concordancia al interior del sintagma nominal como lo atestigua el hecho de que los deícticos tienen una bipartición para masculino/ femenino.

Cuadro 3: Género en deícticos distales

\begin{tabular}{|l|l|c|c|}
\hline \multirow{2}{*}{ Masculino } & & Distal 1 & Distal 2 \\
\cline { 2 - 4 } & Singular & dà & guà \\
\hline \multirow{2}{*}{ Femenino } & Plural & daé & gué \\
\cline { 2 - 4 } & Plurgular & \multirow{2}{*}{ ñàà } & yeà \\
\hline
\end{tabular}

Ejemplos:

düká dà é “imira! ahí está este huito” (genipa)

INJ DIST1+GM huito

guà ya ngué "ahí (está) la canoa"

DIST2+GM G canoa

Base pronominal, CAU Causativo, CÓP Cópula, DIST1 Distal 1, DIST2 Distal 2, G Género, GF Género femenino, GM Género masculino, IN Inanimado, INT Intensificador, aspecto, NOM Nominalizador, derivativo, O INT Objeto interno, O2 Objeto 2 (beneficiario, paciente), PL Plural, TÓP Tópico, 'Terraza tonal, efecto entonativo resultado de procesos de focalización o topicalización. 
ñáà 1̃-yî̃ i o:tá “ésta es gallina”

DIST1+GF 3PFS-ser G gallina

En otras lenguas del área la animacidad es un rasgo central en el paradigma de las formas personales y determina hechos como el uso o no de clasificadores de forma. En tikuna el rasgo de \pm animacidad (cruzada con los tres valores de género) se expresa marginalmente en los demostrativos anafóricos a través de una alternancia tonal que se neutraliza en el femenino+pasado.

Cuadro 4: Animacidad marcada por alternancia tonal cruzada con tres valores de género en demostrativos anafóricos

\begin{tabular}{|c|c|c|c|c|}
\hline \multirow{4}{*}{ 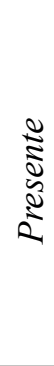 } & \multirow{2}{*}{ 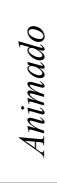 } & Masculino & $\begin{array}{l}\text { yimá ya airú "este perro" } \\
\text { AN+MASC G perro }\end{array}$ & $\mathrm{MB}$ \\
\hline & & Femenino & $\begin{array}{l}\text { ngé:mà i airú "esta perra" } \\
\text { AN+FEM G perro }\end{array}$ & $\mathrm{AB}$ \\
\hline & \multirow{2}{*}{ 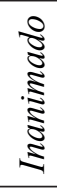 } & Masculino & $\begin{array}{l}\text { yìmà ya nai “ese árbol” } \\
\text { IN+MASC G árbol }\end{array}$ & $\mathrm{BB}$ \\
\hline & & Femenino & $\begin{array}{l}\text { yèmà i üú́ “esa leña” } \\
\text { IN+FEM G leña }\end{array}$ & $\mathrm{BB}$ \\
\hline \multirow{3}{*}{ 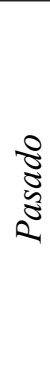 } & \multirow{2}{*}{ 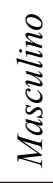 } & Animado & $\begin{array}{l}\text { gumá ga úüne "ese inmortal" } \\
\text { IN+PAS G árbol }\end{array}$ & MA \\
\hline & & Inanimado & $\begin{array}{l}\text { gùmà ga nai “ese árbol” } \\
\text { IN+PAS G árbol }\end{array}$ & $\mathrm{BB}$ \\
\hline & & 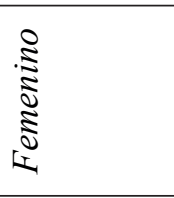 & $\begin{array}{l}\text { yé:mà i üú̉ "esa leña" } \\
\text { IN+FEM G leña } \\
\text { yé:mà i airú “esta perra" } \\
\text { AN+FEM+PAS G perro }\end{array}$ & $\mathbf{A B}$ \\
\hline
\end{tabular}

Al interior del sintagma nominal y en presencia de determinantes cuạntificadores y numerales, la marca de género es un proclítico obligatorio: gúü ya îtú "todos los chontaduros" todos $\mathrm{G}$ chontaduro ta:ré i tàrá "2 machetes"

$2 \mathrm{G}$ machete

wüì ya yátü “1 varón”

$1 \mathrm{G}$ macho

tà:ré i pá:-kü-gü "2 muchachas"

$2 \mathrm{G}$ ser mujer joven-NOM-PL 
wüì ga üǜne "1 ser inmortal"

$1 \mathrm{G}$ inmortal

Otro contexto de obligatoria aparición del proclítico de género es la construcción en la que dos nombres libres están en relación de determinación y forman una lexía estable que puede ser, como en este caso, la denominación de una especie de pez.

uchúmá ya îtú "tipo de piraña"

piraña $\mathrm{G}$ chontaduro (bactris gasipae)

Igualmente ocurre con la determinación de un nombre simple por un nominal derivado (no hay adjetivos puros en esta lengua, las nociones calificativas son verbos intransitivos y para determinar a un nombre deben nominalizarse), sea para constituir una lexía estable (denominación de una especie vegetal), sea para hacer una adjetivación ('perro bravo'):

îtü ya chó:-kü "chontaduro blanco (tipo de chontaduro)"

chontaduro G ser blanco-NOM

airú ya düra-kü "perro bravo"

perro G ser bravo-NOM

Finalmente en hablas de adultos no ribereños subiste una alternancia tonal en nombres libres que sirve para diferenciar el sexo en referentes que tienen una semántica transparente (gallo/gallina). Este mecanismo se puede extender a algunos nominales derivados como aquellos que designan estadios de la vida humana, en este caso, derivados del verbo na-bú: "ser niño, infante" (frente a cada ítem se escriben los tonos fonológicos en mayúscula y la realización tonal fonética en minúsculas):

Cuadro 5: Cambios tonales según el género en palabras con género transparente

\begin{tabular}{|l|l|l|l|}
\hline o:tá “gallina" & MA ---> ma a' & ò:tá “gallo" & BA ---> ab a \\
\hline bù:kü “niño varón” & BM ---> ab m & bú:kü “niña” & A ---> ba m \\
\hline
\end{tabular}

Por establecer si la alternancia tonal masculino/femenino se mantiene sistemáticamente en adjetivos derivados de bases verbales bimoraicas como chó:-kü "blanco", daù-kü "rojo" y otras, lo que implicaría que la concordancia dentro del sintagma nominal tendría mayor alcance. 


\section{Nombres clasificadores: polo gramatical y polo léxico}

Los nombres son ligados (dependientes, inalienables) o libres. Los nombres ligados son principalmente aquello que remite a partes de un todo, típicamente el cuerpo humano y el parentesco (con excepción de órganos sobresalientes y de puntos sobresalientes de las relaciones de alianza):

na-nátü "padre de él"

na-kutứ "pie, orilla"

También son nombres ligados elementos y artefactos cercanos al ámbito personal (la canoa, la hamaca, el collar, los adornos) y las partes de los artefactos, también una serie de conceptos abstractos como na-chígá "rito, historia, significado". Igualmente, una buena parte de los nombres que designan espacios geográficos y espacios sobrenaturales. El término na:-ànè designa una noción amplia de 'cosmos', 'mundo', entendido como el ámbito espacial y temporal del que hacen parte los cursos de agua (na:-tü), los cerros (na:-pü) y otros elementos similares. Este mismo radical nominal -ànè recibe las marcas de persona para designar la tierra de cultivo poseída por alguien, por ejemplo, na-ànè designa 'tierra o chagra de él'. Esta importante diferencia entre nombres ligados que son parte de un todo pero que no son realmente poseíbles y nombres ligados que pueden recibir las marcas posesivas tiende a debilitarse por la pérdida de diferencia fonológica entre los prefijos \{na:- $\}$ para lo no poseíble y \{na-\} para la tercera persona masculina o genérica. Esta indiferenciación de dos morfemas gramaticales responde a un proceso en curso en la lengua que neutralizaría las oposiciones de longitud vocálica (el tono subyacente de ambos prefijos es medio, difiere la realización fonética).

Los nombres libres son típicamente nombres de especies animales o vegetales vistas de manera global, algunos artefactos fabricados o comprados y algunos elementos del mundo natural (piedra, estrella, isla fluvial, un tipo de arcilla, entre otros).

La construcción NOMBRE LIBRE+NOMBRE LIGADO es un mecanismo productivo en la morfosintaxis de esta lengua:

gúré-nátü "padre o dueño del curare" (veneno paralizante)

curare-padre

dé:nè-ménáà "tallo (hueco) de la caña de azúcar"

caña de azúcar-tubular 


\subsection{Nombres incorporados obligatoriamente. Hacia la gramaticalización}

Un subconjunto de los nombres ligados muestra una incipiente gramaticalización, a partir de los siguientes hechos:

\subsubsection{Tamaño y forma-postura}

Los predicados relativos a la dimensión de una entidad "ser grande", "ser pequeño", "ser largo" (vertical o no vertical), especialmente si están referidos a entidades animadas (vegetal, animal, humano), exigen la incorporación del nombre ligado con función de clasificador. Los nombres-clasificadores deben incorporarse obligatoriamente dentro de la palabra verbal y no hay posibilidad de forma alterna en dos palabras, como sí la hay en la mayoría de construcciones con incorporación con otros verbos. Todo nombre ligado ('tubular' o 'caña', 'cabello', 'pie' en los ejemplos del cuadro 6) permite -con ciertos verbos-elegir entre una forma con incorporación y una forma sin incorporación:

Cuadro 6: Comparación de construcciones con y sin incorporación

\begin{tabular}{|c|c|}
\hline Incorporación & Sin incorporación \\
\hline $\begin{array}{l}\text { na-yaura-ménáà “(es) brillante" } \\
\text { 3PMS-ser brillante-tubular }\end{array}$ & $\begin{array}{l}\text { na-ménáà na-yaura "lo tubular (es) brillante" } \\
\text { 3PMS-tubular 3PMS-ser brillante }\end{array}$ \\
\hline $\begin{array}{l}\text { ngĩ-mẽ̀:-yáè “(es) bonito su cabello" } \\
\text { 3PFS-ser bonito-cabello }\end{array}$ & $\begin{array}{l}\text { ngĩ-yáè na-mẽ̀: “(es) bonito su cabello" } \\
\text { 3PFS-cabello 3PS-ser bonito }\end{array}$ \\
\hline $\begin{array}{l}\text { kù-tà:-kùtú “(es) grande tu pie” } \\
\text { 2PS-ser grande-pie }\end{array}$ & $\begin{array}{l}\text { kù-kùtú na-tà: “(es) grande tu pie” } \\
\text { 2PS-pie 3PS-ser grande }\end{array}$ \\
\hline $\begin{array}{l}\text { chà-ü-pátá "fabrico casa" o } \\
\text { "fabrico mi casa" } \\
\text { 1PS-fabricar-casa }\end{array}$ & $\begin{array}{l}\text { na-pátá chà-ú "fabrico casa" o } \\
\text { "fabrico su casa" } \\
\text { 3PS-casa 1PS-fabricar }\end{array}$ \\
\hline
\end{tabular}

Sincrónicamente los elementos que actúan como clasificadores son también nombres, pero no hay en tikuna ningún indicio fonológico (erosión fonológica, fusión tonal, pérdida silábica) que diferencie estas construcciones obligatorias de otras incorporaciones ni de otros usos de estos nombres como nombres plenos. Ejemplos de la incorporación obligatoria -considerada como un indicio de gramaticalización- con los verbos 'ser largo o alto', 'ser pequeño', 'ser grande' referidos a una entidad globalmente considerada (no a sus partes): 
na-má-chanè "es alto"

3PMS-ser largo-vertical

kù-1́:-chanè "(tú) eres pequeño"

2PS-ser pequeño-vertical

kù-má-chanè "(tú) eres alto"

2PS-ser largo-vertical

na-tá:-tà "es grande" (referido a un animal cuadrúpedo por ejemplo)

3PMS- ser grande-lomo

woka na-tá:-tà "la vaca es grande" cf. *woka na-tá:

vaca 3PMS-ser grande-lomo

na-má-má:்̇̀ "es largo"

3PMS-ser grueso-alargado

ãtape na-má-máǜ "la serpiente es larga"

serpiente 3PMS-ser largo-alargado

La verticalidad es la dimensión propia de los árboles altos, de los humanos y de las aves zancudas y puede extenderse a los artefactos que se puedan asimilar a esta postura (postes, edificios, torres). La dimensión horizontal o de 'lomo' se aplica a animales cuadrúpedos, patos, paujiles y opcionalmente a bancos bajos, mesas, superficie de una silla. La dimensión no vertical, no horizontal, clasifica entidades en posición colgado, reptante o alargado; se aplica típicamente a lianas y a serpientes, y opcionalmente a objetos que se les semejen como cuerdas, mangueras, tubos.

La incorporación del nombre inalienable "cuerpo, configuración" permite eludir la obligatoriedad de indicar la forma-postura en los predicados de tamaño con seres animados:

na-tà:-ǘné "su cuerpo es grande"

3PMS- ser grande- cuerpo

Hay pues un subconjunto de nombres ligados que manifiesta un rasgo formal particular al exigirse su incorporación en un tipo de construcción referida a tamaño. Contrastan con el comportamiento de otros nombres ligados con un espectro semántico similar pero con incorporación opcional. Esto ocurre independientemente del carácter animado del referente. 


\subsubsection{Clasificador metereológico}

En esta lengua puede postularse una construcción en la que interviene un nombre-clasificador metereológico. El nombre para 'tierra, cosmos, mundo' es obligatoriamente incorporado en la mayoría de las expresiones que describen el tiempo metereológico:

na-déyù-ànè "hace frío" *na-ànè na-déyù

3PMS-hacer frío-tierra

ni-dùrù-ànè "truena"

3PMS-tronar-tierra

Este nombre \{-ànè $\}$ se encuentra en otros contextos en donde su valor como nombre pleno de amplio semantismo es claro.

Lo expuesto en este apartado sobre la incorporación obligatoria de un subconjunto de nombres ligados \{-chanè -tà -má, -máü, -ànè\} es el hecho que, desde nuestro punto de vista, justifica hablar de clasificación y de nombresclasificadores.

\subsection{El polo léxico Nombre clasificador opcional: Elección del hablante, singularización, definición}

La incorporación es opcional con otras configuraciones de forma o cuerpos (caparazón, compacto redondeado, cubiertas coriáceas) de seres animados e inanimados o artefactos fabricados.

na-tá:-táwè "es grande-cascudo" (referido a una tortuga por ejemplo)

3PMS-ser grande-caparazón

ngáwè a í-ürà-púuìi-üchì-kü "totuma muy pequeña" (refiriéndose al fruto redondeado no a la concavidad del recipiente)

totuma G ser pequeño-ATE-compacto, fruto-INT-NOM

En todos los casos se trata de elementos léxicos que pertenecen a un paradigma abierto y que son importantes como mecanismo en la creación de neologismos. Su semántica abstracta y englobante les confiere un poder clasificatorio. Como ejemplo, na-púiù es un nombre ligado que designa lo compacto, lo que está en forma de paquete, cualquier fruto redondeado, que entra en la formación de nombres como rodilla (literalmente 'la que tiene algo redondeado') y que puede ser usada como base para construir el neologismo para 'balón de fútbol'. 
Listamos algunos ejemplos de otros nombres de forma con una traducción apenas aproximada de su valor semántico en la que hemos incluido algunas ocurrencias típicas:

na-nú́tà "filiforme, largo y delgado, fibra, hilo, lombriz, serpiente delgada"

na-chìnù̀ "laminar, plano, rígido, disco, disco de la luna, cadera, oreja, mesa"

na-párá "soporte, tubérculo, fruto de banana, pierna o pata de animales o de muebles"

na-chípáà "cubierta dura, caparazón, corteza, escama, piel coriácea, concha, envoltura rígida"

La forma-postura (vertical-parado, horizontal-sentado, colgado-reptante) de los referentes es un rasgo privilegiado en la categorización que expresan los clasificadores léxicos o gramaticalizados. Sin embargo, intervienen otros rasgos semánticos globales y abstractos, también con un gran poder clasificatorio, en nociones que cruzan forma, textura, consistencia, materia. A continuación algunos ejemplos de esos nombres ligados, también elementos de paradigmas abiertos:

na-téè "harina,polvo, material triturado o granulado"

ui-téè "harina de yuca, fariña (granulada)"

koka-téè "polvo de coca (polvo muy fino)"

na-chara "materia flexible y blanda, barro, tejido"

ñoé-chara "tela de fibra vegetal, 'yanchama' o 'tururí' (ficus)"

na-chiru-chara "tela para hacer vestidos"

3P-vestidos-tejido

na-mù̀ “masa blanda, puré, pasta, maceración, tierra, semen”

tüè-mù̀ "puré de yuca"

na-tû́û்̃ "líquido en el cual nada o se disuelve algo, líquido amniótico"

chòní-tứû̀ “caldo de pescado"

na-chíù "líquido extracto, zumo"

Los numerales no desencadenan la aparición obligatoria del nombreclasificador. Pareciera más bien que la elección depende de factores de tipo pragmático y de la necesidad de desambiguar o definir un nombre en un contexto comunicativo dado. Cualquiera de las siguientes expresiones puede ser adecuada, dependiendo de que se haga énfasis en la forma de un elemento 
del conjunto o en la del conjunto como tal. Si el contexto es compartido, se prescinde de toda mención a la forma:

wứì-ménáà a pori " 1 cigarrillo"

1-tubular G tabaco

wứì-púiù a pori "1 paquete de cigarrillos"

1-compacto $\mathrm{G}$ tabaco

pori-ménáà "tabaco enrollado"

tabaco-tubular

wưì a pori "1 cigarrillo"

$1 \mathrm{G}$ tabaco

\subsection{Funciones de los nombres clasificadores}

Como ya han señalado otros lingüistas para algunas lenguas amazónicas en las que se puede rastrear el origen léxico y se detecta la presencia simultánea del clasificador como nombre- la función principal de estas formas está del lado de la derivación, la composición y también en las operaciones pragmáticas a través de las cuales el hablante define, precisa, singulariza, según necesidades del contexto previo de comunicación. Sobre la función de la construcción con los nombres ligados en hup, se subraya la función de individuación de la construcción con nombres ligados $^{12}$. En el uitoto ${ }^{13}$ se dice que la función más frecuente y sobresaliente de los clasificadores es especificar el perfil físico de un referente particular en una situación particular.

\subsubsection{Derivación y composición}

Los nombres ligados, tanto aquellos que muestran un grado parcial de gramaticalización como los que son elementos puramente léxicos, son claves para diferenciar los valores genéricos (especie vegetal, referencia general al nombre, carácter no definido ni singularizado de una entidad) y los valores más específicos, definidos, singularizados o parciales de la entidad.

îtû́ "chontaduro" (nombre de la especie o de una parte si ya se sabe de qué parte se habla)

\footnotetext{
${ }^{12}$ Epps, Patience. "Birth of a Noun Classification System" En: Language Endargement and Endangered Languages. Linguistic and Anthropologicial Studies with special emphasis on the Language and Cultures of the Andean-Amazonian border Area, editado por Leo Wetzels. (Leiden: CNWS Publications, Vol. 154, 2007), 109.

${ }^{13}$ Petersen, Gabriele. "Nominal Classification in Uitoto" International Journal of American Linguistics, vol. 73, N. 4 (2007), 408-409.
} 
îtú́-pứủ "fruto del chontaduro individualizado, fuera del racimo"

îtû́-réè "gajo del racimo de frutos de chontaduro"

También puede considerarse que son elementos claves en la lexicogenia, para la formación de nombres compuestos y para la creación de neologismos:

bóò-chìnù̀ "luna creciente" (fase previa a la luna llena)

ser esférico- lámina

na-pà-púuù "ovillo de fibra para tejer hamaca"

3PMS-hamaca-compacto

bóò-púiù "balón de fútbol" (esfera compacta)

ser esférico-compacto, paquete

En el caso tikuna nos parece interesante destacar tres nombres ligados de amplio semantismo, cuya acepción más básica la encontraríamos en el ámbito de los ecosistemas, desde donde parecen extenderse para designar realidades sociológicas o nociones cercanas a la cuantificación.

El primero de los nombres es nombre \{-chíkúu que cuando determina a otro nombre, designa lo que está lejos del prototipo, 'pseudo'. Su sentido básico es el de antiguo terreno cultivado o rastrojo que se abandona.

ĩ-chíkú́ "antiguo terreno cultivado, morada abandonada"

morada-seudo

na-chíkû́ "rastrojo de él"

3PMS- rastrojo

Con nombres de especies vegetales permite obtener una nueva entrada léxica. Puede tratarse de una especie similar en aspecto caso en el cual tendríamos un procedimiento metafórico o metonímico.

poí-chíkû́ "platanillo, heliconia"

plátano-rastrojo

En otros casos, la composición NOMBRE LIBRE+NOMBRE LIGADO puede designar especies que usualmente son cultivadas en la chagra, pero que crecen también fuera del campo de cultivo de manera silvestre, en los rastrojos abandonados o en lugares no habitados y que los tikunas consideran como especies 'ordinarias'. Este nombre también permite referirse a comportamientos sociales lejanos al prototipo. 
korí-chíkû́ "mestizo o indio con comportamiento de blanco" blanco-seudo

El segundo elemento que nos interesa destacar es un nombre que designa colectividades o agrupaciones (racimos, manadas, bandadas; grupos humanos de niños o de mujeres, que son quienes normalmente andan en grupos).

poí-tà "bananas en racimo"

banana-agrupación

ènè-tà "loritos en bandada"

loritos-agrupación

bú:-ã-tà "niños en grupo"

ser niño-NOM-agrupación

En lenguas con clasificadores gramaticalizados como el sáliba ${ }^{14}$, la designación de colectivos parece plantear un problema para la lógica del sistema de clases por la diversidad e irregularidad de los procedimientos morfosintácticos usados para los inanimados. En otras lenguas unos pocos colectivos permiten reagrupar los nombres que habían sido individualizados previamente a través de un extenso paradigma de clases o clasificadores: en lenguas de la familia Tukano, una única marca agrupa a todos los inanimados no singulares; mientras que masculino y femenino animado se unifican en una única marca de animado plural ${ }^{15}$.

El tercer nombre designa exclusivamente ciertas agrupaciones de vegetales que crecen en un mismo espacio, típicamente las agrupaciones de palmas que crecen juntas y que son puntos importantes de referencia en la topografía selvática (los 'manchales' de canangucho, de chontaduro, de asaí, de milpeso). El nombre común para designar el monte o selva, opuesto a la aldea y a la chagra, es el término genérico para 'árbol' y el nombre colectivo para manchal o agrupación vegetal.

nai-nékú́ "selva, arboleda"

árbol-manchal

témá-nékú "cananguchal”

palmera Mauritia-manchal

\footnotetext{
${ }^{14}$ Estrada, Hortensia. La lengua Sáliba. (Bogotá: Colcultura- Tercer Mundo editores, 1996): 76-80.

${ }^{15}$ Gómez-Imbert, “Tukanoan Nominal Classification”, 408.
} 
Igualmente el término se encuentra en otras agrupaciones que caracterizan ecosistemas particulares. Terrenos arenosos caracterizados por carencia de árboles y de vegetación alta, son denominados en español regional 'varillal' (porque crecen 'varas' y no árboles grandes). El tikuna hace una construcción que literalmente se analizaría como 'sitios de colectividades vegetales infantiles':

bú:-nékǘ "varillal"

ser niño-manchal

Creemos que las derivaciones que permiten estos nombres, relacionadas con lo prototípico y con las colectividades o agrupaciones, serían elementos propicios para un proceso de gramaticalización.

\subsubsection{Anáfora}

En el nivel textual, los nombres-clasificadores gramaticalizados o no, permiten la continuidad de la referencia y la retoma o ampliación de los argumentos previamente presentados sin estar sometidos a las restricciones de la concordancia. Como deja ver el ejemplo, la palabra para 'ovillo' aparece en primer lugar incorporada, también se incorpora el nombre ligado 'borde'; luego aparece con el nombre 'hamaca' para designar algo que se considera como parte de la misma (el ovillo de fibra vegetal para tejerla).

yiémà-rü tùu ta-na-û́-pú́ù̀,

ésta-TÓP espina 1PP-OINT-hacer-compacto

"Con esta aguja hacemos el ovillo,

na-kà niĩ ta-na-úi-chawenáà i napà,

3P-O2 CÓP G hamaca 1PP-OINT-hacer-borde G hamaca

de eso es que hacemos el borde de la hamaca,

ta-na-û́-púiù,

1PP-OINT-hacer-compacto

hacemos el ovillo,

napa-púiù niĩ

hamaca-compacto CÓP

es ovillo de hamaca"

La función textual y pragmática de los nombres ligados con valor clasificatorio es un elemento clave para caracterizar los géneros de la oralidad, tanto los que implican elaboración discursiva (mitos, palabra de consejo) como los que se dan en el habla cotidiana. Este tema no está explorado aún y constituye el objeto de una tarea en curso. 


\subsection{Aproximación al contenido de los nombres-clasificadores}

Sobrepasa las intenciones y posibilidades de este artículo abordar el contenido cultural y cognitivo que está tras la expresión y el uso de los clasificadores. Señalamos algunos elementos que pueden ser útiles para un análisis ulterior, más centrado en la semántica y en la pragmática.

En tikuna las designaciones del 'cuerpo' de las plantas serían las ocurrencias prototípicas y aquello que mejor nos permitiría acercarnos al plano conceptual. Sin embargo esta idea de la mutua implicación entre cuerpo y naturaleza en el marco de la cultura, es algo que ameritaría una extensa discusión como la que han emprendido etnólogos entre otros, sobre los miraña ${ }^{16} \mathrm{y}$ los tikuna ${ }^{17}$. Hablar de una extensión semántica o de una metáfora entre cuerpo y naturaleza parece una simplificación excesiva. De hecho el cuerpo -humano, animal, vegetal, cósmico- es la sede de una conceptualización mutuamente imbricada que se expresa en una terminología compartida. La pregnancia de esta ideología sobre el mundo y las cosas es tal que no es sólo un hecho léxico sobresaliente, sino que permea la gramática como lo muestran los procesos visibles en los diferentes grados de codificación-obligatoriedad que tienen estas formas en lenguas de la región. Aunque tipológicamente las lenguas difieran de maneras importantes (obligatoria concordancia o no en el nivel de la oración, pertenencia de los clasificadores a un paradigma gramatical cerrado o inventario abierto de nombres, composición o flexión, clasificación de forma sólo con inanimados o también con animados) en términos de contenido hay similaridades entre lenguas y familias no emparentadas. Estas similaridades remiten también a un universo cultural que ordena un ecosistema compartido.

Algunas observaciones conclusivas sobre este apartado:

- En el ámbito de las denominaciones vegetales están las ocurrencias 'prototípicas'. Algunas son definitivamente léxicas, constreñidas a expresar un nombre inalienable en el contexto de la determinación. Aparecen en el discurso cuando ya se ha hablado de la entidad (una caña, un árbol, un bejuco, una pepa) y se quiere agregar una información adicional de las características de la misma (función anafórica). Varios autores que trabajan el tema señalan en sus análisis y en sus ejemplos la centralidad de las plantas y los ecosistemas en el entendimiento de las nociones que vehiculan los clasificadores.

\footnotetext{
${ }^{16}$ Karadimas, Dimitri. La raison du corps. Idéologie du corps et representation de l'environement chez les Miraña d'Amazonie colombienne. Colección Langues et societés d'Amérique Traditionnelle. (Paris: Editions Peeters-SELAF, 2005).

${ }^{17}$ Goulard, Jean Pierre. "Les genres du corps. Conception de la personne chez les ticuna de la haute Amazonie". (Tesis doctoral, École de Hautes Études en Sciences Sociales, Paris, 1998).
} 
- En predicación de cualificación, con verbos referentes a tamaño o a textura es indispensable indicar la forma y la postura (tres valores: vertical erguido, horizontal sobre soportes, alargado reptante) de una entidad animada. Estos casos, más el clasificador 'atmosférico', configuran un comportamiento gramaticalizado.

- No es pertinente hablar de 'extensión semántica' pues se postula que estos nombres son conceptos abstractos de forma, función, agrupación, cuyas ocurrencias típicas están en el ámbito de la denominación de partes vegetal, animal, humana, de artefactos y del espacio configurado culturalmente (la casa, la chagra, la selva).

- Un subconjunto de nombres ligados que se incorpora obligatoriamente tiene gran poder clasificatorio, son nombres-concepto que no están limitados a las plantas o al cuerpo humano.

- Otros términos de partes de una configuración pertenecen a un paradigma realmente abierto y con referentes limitados y 'concretos' en el que están designaciones específicas de los 'cuerpos' vegetales, animales, humanos, de espacios (chagra, topografía, maloca y casa), de objetos (canoa, otros). Tienen así un espectro semántico más restringido a un ámbito particular. Pertenecen al mismo conjunto morfosintáctico de nombres ligados, pero no a la misma agrupación semántica, pues no son rótulos clasificatorios importantes.

\section{El tikuna en los sistemas de clasificación del Noroeste Amazónico, comentarios preliminares}

Una fuente importante de los sistemas clasificación nominal estaría en el léxico, desde los 'términos de clase'. Por otra parte, en tikuna la primera categorización de los nombres - tan importante como la de género- es la división entre libres y ligados, una categorización con importantes correlatos semánticos, ambientales y culturales. La referencia de los nombres ligados es menos específica que la de los nombres libres, lo que los hace aptos para cumplir funciones análogas a las de los 'verdaderos' clasificadores de otras lenguas

En tikuna el género establece una clasificación exhaustiva de los nombres, está claramente gramaticalizado y sus contextos de aparición son obligatorios, es por lo tanto un sistema que cubre todos los nombres, incluyendo los que se obtienen por derivación. A diferencia de otras lenguas del área, el género y los clasificadores -o seudo-clasificadores- son dos sistemas independientes. El carácter animado/inanimado se expresa -en otras lenguas del área- en las marcas concordantes de clase, cruzadas con el carácter humano/no humano, femenino/masculino y colectivo. Estos mismos rasgos están presentes en el sistema nominal del tikuna pero están disociados, pues los rasgos del género 
son presente (masculino/femenino) y pasado, mientras que el carácter animado/ inanimado se expresa marginalmente en un paradigma de demostrativos.

En tikuna el género presenta la particularidad de cruzarse con una categoría de tiempo-modalidad (¿interpretable también como distancia-visibilidad?). La clasificación por su parte, está relacionada fundamentalmente con hechos de tipo léxico ligados a la composición, a la determinación nominal y sobre todo a la incorporación. Sólo los nombres ligados entran en compuestos en los que el resultado final es de tipo clasificatorio. No hay señales de 'erosión' fonológica que permitan deducir que el nombre ligado está en vías de convertirse en un morfema gramatical, como ocurre en otras lenguas, en las cuales se ha rastreado el origen léxico del clasificador. Contrasta también con el uitoto, lengua en la cual es posible elidir sílabas en uno de los compuestos (y formar 'proformas'), una señal importante del grado de integración del clasificador en lo que podría considerarse una señal de gramaticalización incipiente ${ }^{18}$. Lo que encontramos en tikuna se parecería más a los hechos descritos para el yuhup ${ }^{19}$ y para el hup ${ }^{20}$, en los cuales es clara la plena vigencia de los nombres que intervienen en la estrategia de clasificación nominal. Parece necesario hacer una ampliación tipológica exhaustiva para el Noroeste Amazónico que incluya lenguas como el sáliba y el andoke, pertenecientes a estirpes no consideradas hasta el momento. Cada una de las lenguas del área ya descritas representa un punto en la gramaticalización de los clasificadores. Sistematizar estos hechos sería de mucho interés para ubicar lenguas como el tikuna en las cuales hay una gramaticalización parcial. Aún se requiere ampliar la comparación detallada de una mayor cantidad y variedad genética de las diversas lenguas presentes en esta región amazónica.

\section{Anexo}

Familias y lenguas en las que se ha estudiado el fenómeno de clasificación nominal en el Noroeste amazónico y Orinoquia de Colombia:

1. Arawak: achagua (Ach), baniwa del Isana (Bw), kurripako (Kr), tariana (Tr),

2. Sáliba-Piaroa: sáliba $(\mathrm{Sb})$

3. Makú: hup (Hu), yuhup (Yu)

4. Tukano Oriental tatuyo (Tt), tanimuka ${ }^{21}(\mathrm{Tn})$

\footnotetext{
${ }^{18}$ Petersen, Nominal Classification in Uitoto, 402.

${ }^{19}$ Ospina, A. M. "Clasificación nominal en Yujup” Amérindia 29/30. (Paris: A.E.A. 2006), 180.

${ }^{20}$ Epps, Epps, "Birth of a Noun Classification System", 108.

${ }^{21}$ Eraso, Natalia "De lo léxico a lo gramatical: sistemas de clasificación nominal en tanimuca" Amérindia 29/30 (2006): 161-178. Paris: Centre National de la Recherche Scientifique- A.E.A.
} 
5. Andoke: andoke (Ad)

6. Uitoto: uitoto (4 dialectos) (Ui), okaina ${ }^{22}(\mathrm{Ok})$

7. Bora-Miraña: bora (Bo), miraña (Mi), muinane ${ }^{23}(\mathrm{Mn})$

8. Yurí-Tikuna: tikuna $(\mathrm{Tk})$

9. Peva-Yagua: yagua (Yg)

Otras lenguas en las que se menciona la presencia de clasificadores:

Tukano Oriental: barasana $(\mathrm{Bs})$, carapana $(\mathrm{Cr})$, macuna $(\mathrm{Mc})$, bará $(\mathrm{Br})$, tuyuca (Ty) y tanimuca $(\mathrm{Tn})^{24}$; cubeo $(\mathrm{Cb})^{25}$, yurutí $(\mathrm{Yr})^{26}$, wanano $(\mathrm{Wn})^{27}$.

Tukano Occidental: koreguaje $(\mathrm{Kg})^{28}$

Mapa elaborado por M. E. Montes. Croquis de 'Colombia Física' ${ }^{29}$. Ubicaciones tomadas de estudio sobre lenguas amazónicas ${ }^{30}$ y complementado con ubicaciones del mapa publicado por el Instituto Caro y Cuervo ${ }^{31}$.

\footnotetext{
${ }^{22}$ Estudio de Doris Fagua en este volumen.

${ }^{23}$ Estudio de Consuelo Vengoechea en este volumen.

${ }^{24}$ Gómez-Imbert, Elsa "Las lenguas del Pirá-Paraná” En: Lenguas indígenas de Colombia, una visión descriptiva, González y Rodríguez (Editoras). (Bogotá: Instituto Caro y Cuervo, 2000): 338 .

${ }^{25}$ Hollinger, Cari, Criswell, Linda y Morse, Nancy "El cubeo" En: Lenguas indígenas de Colombia, una visión descriptiva, González y Rodríguez (Editoras). (Bogotá: Instituto Caro y Cuervo, 2000): 360-361.

${ }^{26}$ Kinch, Rodney y Kinch, Pamela "El yurutí” En: Lenguas indígenas de Colombia, una visión descriptiva, González y Rodríguez (Editoras). (Bogotá: Instituto Caro y Cuervo, 2000): 475.

${ }^{27}$ Waltz, Carolyn y Waltz, Nathan "El wanano" Lenguas indígenas de Colombia, una visión descriptiva, González y Rodríguez (Editoras). (Bogotá: Instituto Caro y Cuervo, 2000):458460.

${ }^{28}$ Dupont, Carlos "Clasificación nominal en la lengua koreguaje" en Forma y Función, 4 (1989): 35-46. Bogotá: Departamento de Lingüística de la Universidad Nacional de Colombia.

${ }^{29}$ Instituto Geográfico Agostini Novara. Atlas de Colombia y el mundo. (Bogotá: Panamericana Editorial, 2010): 96.

${ }^{30}$ Centro Colombiano de Estudios de Lenguas Aborígenes CCELA. "Lenguas indígenas de la Amazonia colombiana” En: As línguas amazónicas hoje, Queixalós y Renault-Lescure (Organizadores). Sao Paulo: Instituto Socioambiental, 2000.

${ }^{31}$ Queixalós, F. y Rodríguez, M. L. "Quinta Región: Orinoquia” En Lenguas indígenas de Colombia. Una visión descriptiva, González, M.S. y Rodríguez, M.L. (coordinadoras), Bogotá: Instituto Caro y Cuervo, 2000.
} 
Familias y lenguas en las que se ha estudiado el fenómeno de clasificación nominal en el Noroeste amazónico y Orinoquia de Colombia

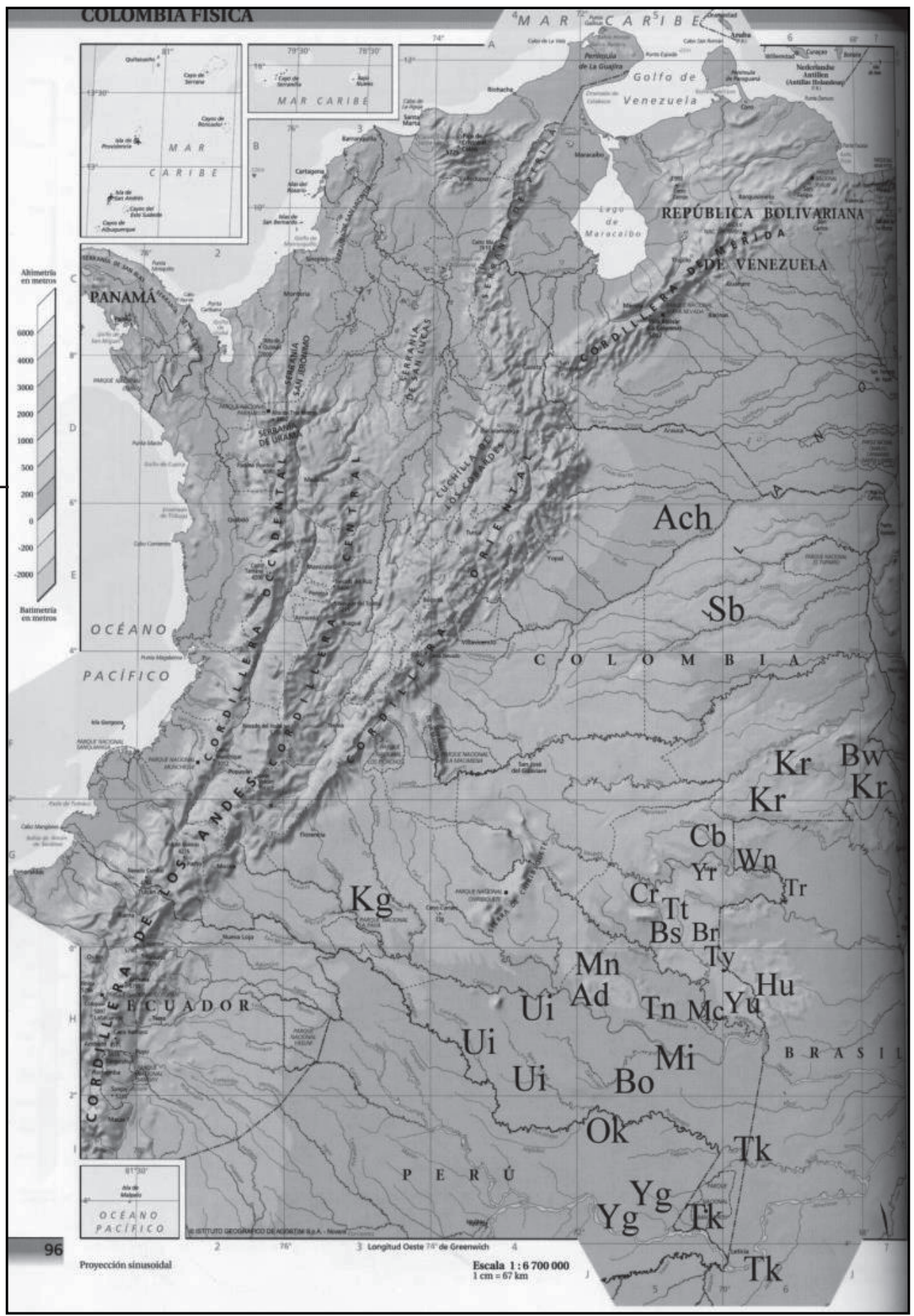




\section{Referencias}

Aikhenvald, Alexandra "Classifiers in multiple environments: baniwa of Icana/ Kurripako-A North Arawak perspective" International Journal of American Linguistics, vol. 73, N. 4 (2007): 475-500. The University of Chicago Press.

Aikhenvald, Alexandra. Classifiers: A Typology of Noun Categorization Devices. Oxford: Oxford University Press, 2000.

Anderson, Lambert "The structure and distribution of Ticuna independent clauses" Linguistics 20 (1966), París: Mouton \& Co.

Anderson, Lambert "Ticuna vowels with special regard to the system of five tonemes” En: Publicacacões Alvusas do Museu Nacional. Rio de Janeiro, 1959.

Carvalho, Fernando Orphão de "On the genetic kinship of the languages Tikúna and Yur'”" Revista Brasileira de Linguística Antropológica 1, Universidad de Brasilia-LALI (2009): 247-268.

Centro Colombiano de Estudios de Lenguas Aborígenes CCELA. "Lenguas indígenas de la Amazonia colombiana" En As línguas amazónicas hoje, organizado por Queixalós y Renault-Lescure. Sao Paulo: Instituto Socioambiental, 2000.

Craig, Colette. “A morphosyntactic typology of classifiers.” En Systems of Nominal Classification, editado por Günther Senft, 50-92, Cambridge: Cambridge University Press, 2000.

Dupont, Carlos “Clasificación nominal en la lengua koreguaje” en Forma y Función, 4 (1989):35-46. Bogotá: Departamento de Lingüística de la Universidad Nacional de Colombia.

Epps, Patience. "Birth of a Noun Classification System" En: Language Endargement and Endangered Languages. Linguistic and Anthropologicial Studies with special emphasis on the Language and Cultures of the Andean-Amazonian border Area, editado por Leo Wetzels, 107-126. Leiden: CNWS Publications, Vol. 154, 2007.

Eraso, Natalia "De lo léxico a lo gramatical: sistemas de clasificación nominal en tanimuca" Amérindia 29/30 (2006): 161-178. Paris: Centre National de la Recherche Scientifique- A.E.A.

Estrada, Hortensia. La lengua Sáliba. Bogotá: Colcultura- Tercer Mundo editores, 1996.

Gómez-Imbert, Elsa “When animals become 'rounded' and 'feminine': Conceptual categories and linguistic classification in a multilingual setting" En Rethinking Linguistic Relativity, editado por Gumperz \& Levinsohn. Cambridge: Cambridge University Press, 1996.

Gómez-Imbert, Elsa "Las lenguas del Pirá-Paraná" En: Lenguas indígenas de Colombia, una visión descriptiva, editado por M.Stella González y M. Luisa Rodríguez. Bogotá: Instituto Caro y Cuervo, 2000. 
Gómez-Imbert, Elsa. «De la forme et du sens dans la classification nominale en tatuyo». Tesis doctoral, Universidad de la Sorbona, Paris, 1982.

Gómez-Imbert, Elsa. "Tukanoan Nominal Classification" En: Language Endargement and Endangered Languages. Linguistic and Anthropologicial Studies with special emphasis on the Language and Cultures of the AndeanAmazonian border Area, editado por Leo Wetzels, 401-428. Leiden: CNWS Publications, 2007.

Goulard, Jean Pierre y Montes, María Emilia “Los Yurí/Juri-Tikuna, en el complejo socio-lingüistico del noroeste amazónico" LIAMES Linguas Indígenas Americanas, vol. 13 (2013):7-65. Campinas: Instituto de Estudios del Lenguaje I.E.L.

Goulard, Jean Pierre. "Les genres du corps. Conception de la personne chez les ticuna de la haute Amazonie". Tesis doctoral, École de Hautes Études en Sciences Sociales, Paris, 1998.

Hollinger, Cari, Criswell, Linda y Morse, Nancy "El cubeo” En: Lenguas indígenas de Colombia, una visión descriptiva, editado por González y Rodríguez. Bogotá: Instituto Caro y Cuervo, 2000.

Instituto Geográfico Agostini Novara. Atlas de Colombia y el mundo. Bogotá: Panamericana Editorial, 2010.

Karadimas, Dimitri. La raison du corps. Idéologie du corps et representation de l'environement chez les Miraña d'Amazonie colombienne. Colección Langues et societés d'Amérique Traditionnelle. Paris: Editions Peeters-SELAF, 2005.

Kinch, Rodney y Kinch, Pamela "El yurutí” En: Lenguas indígenas de Colombia, una visión descriptiva, editado por González y Rodríguez. Bogotá: Instituto Caro y Cuervo, 2000.

Landaburu, Jon. «Quand le nombre est un genre: en Andoke et en quelques autres Langues amérindiennes de Colombie». En: Faites de Langues 2: 145-159. Paris: Presses Universitaires de France, 1993.

Landaburu, Jon. "La lengua andoke" En Lenguas indígenas de Colombia. Una visión descriptiva, editado por González, M. Stella y Rodríguez, M. Luisa, 275288. Bogotá: Instituto Caro y Cuervo, 2000.

Meléndez, Miguel A. La lengua achagua. Estudio gramatical. Colección Lenguas Aborígenes de Colombia, Descripciones, 11. Bogotá: Universidad de Los Andes-CESO-CCELA, 1998.

Montes Rodríguez, M. Emilia. Morfosintaxis de la lengua tikuna. Colección Lenguas Aborígenes de Colombia, Descripciones, 15. Bogotá: Universidad de Los Andes-CESO-CCELA, 2004.

Nimuendaju, Kurt. The Tukuna. Berkeley: University of California Press, 1952

Ospina, A. M. "Clasificación nominal en Yujup" Amérindia 29/30. Paris: Centre National de la Recherche Scientifique -A.E.A. (2006):179-194. 
Palmer, Gary. Lingüística cultural. Madrid: Alianza Editorial, 2000. (Originalmente publicada por The University of Texas Press, 1996).

Payne, Doris "Source of the Yagua Nominal Classification System" International Journal of American Linguistics vol. 73, N. 4 (2007): 447-474. The University of Chicago Press.

Petersen, Gabriele. "Nominal Classification in Uitoto" International Journal of American Linguistics, vol. 73, N. 4 (2007): 389-409. The University of Chicago Press.

Queixalós, F. y Rodríguez, M. L. "Quinta Región: Orinoquia” En Lenguas indígenas de Colombia. Una visión descriptiva, editado por González, M.Stella y Rodríguez, M.Luisa. Bogotá: Instituto Caro y Cuervo, 2000.

Rivet, Paul. “Affinités du Tikuna”. Journal de la Societé des Américanistes. Tomo IX: 83-110. Paris, 1912.

Seifart, Frank \& Payne, Doris. "Nominal Classification in the North West Amazon: Issues in Areal Diffusion and Typological Characterization." International Journal of American Linguistics, vol. 73, N. 4 (2007): 381-388. The University of Chicago Press.

Seifart, Frank "The Prehistory of Noun Classification in Witotoan Languages" International Journal of American Linguistics vol. 73, N. 4 (2007): 411-446. The University of Chicago Press.

Waltz, Carolyn y Waltz, Nathan "El wanano" Lenguas indígenas de Colombia, una visión descriptiva, editado por M. Stella González y M. Luisa Rodríguez. Bogotá: Instituto Caro y Cuervo, 2000.

Data recebimento: $12 / 02 / 2014$.

Data aceite: 18/04/2014. 